\title{
On the null origin of the ambitwistor string
}

\author{
Eduardo Casali $^{a}$ and Piotr Tourkine ${ }^{b}$ \\ ${ }^{a}$ Mathematical Institute, University of Oxford, \\ Woodstock Road, Oxford, OX2 6GG U.K. \\ ${ }^{b}$ Department of Applied Mathematics and Theoretical Physics, \\ Wilberforce Road, Cambridge, CB3 OWA U.K. \\ E-mail: casali@maths.ox.ac.uk, pt373@cam.ac.uk
}

ABSTRACT: In this paper we present the null string origin of the ambitwistor string. Classically, the null string is the tensionless limit of string theory, and so too is the ambitwistor string. Both have as constraint algebra the Galilean Conformal Algebra in two dimensions. But something interesting happens in the quantum theory since there is an ambiguity in quantizing the null string. We show that, given a particular choice of quantization scheme and a particular gauge, the null string coincides with the ambitwistor string both classically and quantum mechanically. We also show that the same holds for the spinning versions of the null string and ambitwistor string. With these results we clarify the relationship between the ambitwistor string, the null string, the usual string and the Hohm-SiegelZwiebach theory.

Keywords: Conformal Field Theory, Scattering Amplitudes, Superstrings and Heterotic Strings

ARXIV EPRINT: 1606.05636 


\section{Contents}

1 Introduction 1

2 The tensionless limit 3

2.1 Classical action 3

2.2 Constraint algebra 5

$\begin{array}{lll}2.3 & \text { Quantization and spinning string } & 7\end{array}$

$\begin{array}{lll}3 & \text { The ambitwistor string } & 10\end{array}$

$\begin{array}{lll}3.1 & \text { The ambitwistor string action } & 10\end{array}$

$\begin{array}{lll}3.2 & \text { Residual gauge symmetry } & 12\end{array}$

\begin{tabular}{ll}
3.3 & Equations of motion in the second order form \\
\hline
\end{tabular}

$\begin{array}{ll}3.4 & \text { Equations of motion in the first order form } \\ \end{array}$

4 Relation to tensionful strings $\quad 14$

$\begin{array}{ll}4.1 \text { The bosonic string } & 14\end{array}$

$\begin{array}{lll}4.2 & \text { The spinning string } & 17\end{array}$

$\begin{array}{llr}5 & \text { Discussion } & 18\end{array}$

\section{Introduction}

The Cachazo-He-Yuan (CHY) formulae [1-5] are a remarkable set of formulae, expressing the tree-level scattering amplitudes of a variety of theories (scalar, gauge, gravity) as worldsheet integrals localized onto the solutions of the so-called scattering equations. The Mason-Skinner ambitwistor string [6, 7] gave a rationale for these formulae as a holomorphic string subjected to the constraint $P(z)^{2}=0$, which enforces the scattering equations. ${ }^{1}$ This stringy origin allowed the CHY formalism to be extended in several directions [8-10], including, very interestingly, loops [11-14].

So far, all the theories describable in this formalism turned out to be related to compactifications of type II supergravity or super-Yang-Mills. It is therefore natural to expect that the ambitwistor string could correspond to some field theory limit, i.e. $\alpha^{\prime} \rightarrow 0$, of string theory.

However, the scattering equations are also famously known to govern the dynamics of the opposite limit of string theory, the Gross-Mende limit, where the tension $T=1 /\left(2 \pi \alpha^{\prime}\right)$ goes to zero $[15,16]$. How then could these two limits have anything in common?

In this paper, we argue that this paradox is clarified when the ambitwistor string is seen as a tensionless string. By the end of the 80 's $[17,18]$ it was already noticed that null

\footnotetext{
${ }^{1} P^{\mu}(z)$ is the momentum of the string.
} 
strings [19], or tensionless strings, possess a quantization ambiguity that can lead to two very different theories. The first one is a higher-spin like theory [20], in accordance with the high energy picture of [21]. The other is a theory with a truncated spectrum that contains only a few states. In the case of the RNS - or spinning - null string, this spectrum was observed to be that of type II supergravity in [17]. We review these constructions in this paper and relate them to the ambitwistor string and the scattering equations.

In the tensionless limit, the classical tensionful string constraint $P^{2}+T^{2}\left(\partial_{\sigma} X\right)^{2}=0$, reduces to $P^{2}=0$, therefore it seems natural to believe that both the Gross-Mende limit and the ambitwistor string have the scattering equations at the heart of their dynamics. This happens to be true even though, as well explain below, they are different quantum theories with Fock-spaces built from different vacua. In order for the reader to appreciate this point already now, we give below the definitions of the two different vacua of the quantized tensionless string in terms of the Fourier modes of the momentum and coordinate fields $P^{\mu}$ and $X^{\mu}$;

$$
p_{n}^{\mu}|0\rangle_{H S}=0, \quad \forall n \in \mathbb{Z} \quad \text { vs } \quad\left\{\begin{array}{l}
x_{n}^{\mu}|0\rangle_{A}=0 \\
p_{n}^{\mu}|0\rangle_{A}=0
\end{array} \quad \forall n>0,\right.
$$

where the indices $H S$ and $A$ stand for "higher-spin like" and "ambitwistor", respectively. A significant part of this paper is concerned with a study of the various relationships between these theories, at the classical and quantum level.

Interestingly, the constraint algebra of the tensionless string has been studied recently in particular in the context of the flat holography, and is nowadays known as the Galilean Conformal Algebra [22, 23]. This algebra usually appears from the non-relativistic limit of a conformal field theory, but there exists an interesting coincidence in two dimensions. Two different contractions of the Virasoro algebra, a non-relativistic one where the worldsheet speed of light goes to infinity, and a ultra-relativistic one where it goes to zero, give isomorphic algebras. One could therefore expect that these two limits should, naively, correspond to the two different tensionless theories. But this is not necessarily the case. In fact, both limits give locally gauge-equivalent versions of the same theory. Interestingly, there is yet another gauge choice which wasn't considered before and will give rise to the ambitwistor string. This choice of gauge is crucial in allowing us to identify one of the null string constraint with the holomorphic stress tensor of a chiral conformal field theory.

The ambitwistor string is therefore not the limit of the usual string at the quantum level, but rather the limit of an alternatively quantized string as will be expanded below. This quantization scheme was also used by Siegel in [24], where it was referred to as a 'change of boundary conditions'.

In the following sections we review the classical and quantum aspects of the tensionless or null string, based on older results in the literature. We then reinterpret the ambitwistor string as a gauge-choice of the null string, clarifying some of its geometrical aspects regarding the interplay between its intrinsically degenerate metric and emergent light-cone structure. The relationship with the usual tensionful string is tackled next, where we relate it to a twisted quantization of the string and show how the normal ordering constant is 


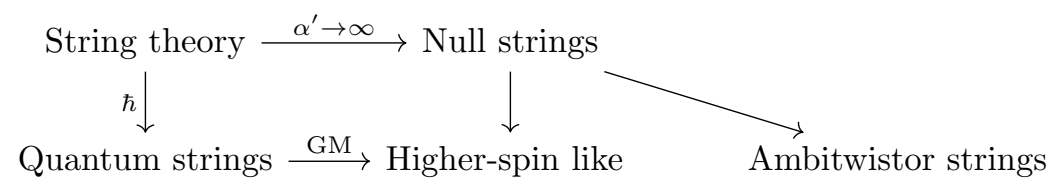

Figure 1. Diagram that illustrates the ambiguity of the order between the limit $\alpha^{\prime} \rightarrow \infty$ or Gross-Mende limit and quantization.

affected by such quantization scheme. The main message of our work is summarized in figure 1.

One of the most interesting applications of our work is that it should pave the way to a more complete understanding of the moduli problem of the ambitwistor string, following in particular [25]. This should allow a geometrical determination of the integration cycle at loop-level [26], something that cannot be done from purely conformal field theoretic considerations. This would also clarify the question of modular invariance in these theories.

\section{The tensionless limit}

The tensionless limit of the bosonic string has been studied in the past from a variety of points of view. Here we summarize the parts of the literature relevant to the ambitwistor string.

\subsection{Classical action}

We start with the Nambu-Goto action

$$
S_{\mathrm{NG}}=-T \int_{M} d^{2} \xi \sqrt{-g},
$$

where $T$ is the tension, $\xi^{\alpha}=(\tau, \sigma)$ for $\alpha=0,1$ are the coordinates on the worldsheet $M$, and $g=\operatorname{det} g_{\alpha \beta}$ where

$$
g_{\alpha \beta}=\frac{\partial X^{\mu}}{\partial \xi^{\alpha}} \frac{\partial X^{\nu}}{\partial \xi^{\beta}} \eta_{\mu \nu}
$$

is the pull-back of the space-time metric to the worldsheet. We take the target space metric $\eta_{\mu \nu}=\operatorname{Diag}(-,+, \ldots,+)$ to be the usual flat metric on Minkowski space. We use standard periodicity $\sigma+2 \pi \simeq \sigma$, so the worldsheet is a cylinder. In this paper we only deal with the closed string. There are tensionless versions of the open string, see [27] in particular, but their geometry is not fully understood, and we leave their connection to a potential open ambitwistor string to future study.

In this action, taking $T=0$ directly is not meaningful. So, as in the relativistic particle case, one should take a detour and either introduce auxiliary fields, as in [17, 28], or go to the Hamiltonian formulation. In both approaches, the tension $T$ appears linearly in the resulting action and the limit is trivial. Here, we follow the latter, as in [29]. From the action (2.1) we obtain canonical momenta

$$
P_{\mu}=T \sqrt{-g} g^{\alpha 0} \partial_{\alpha} X_{\mu}
$$


that obey the following first class constraints

$$
\begin{aligned}
P^{2}+T^{2} g g^{00} & =0, \\
P \cdot X^{\prime} & =0 .
\end{aligned}
$$

We use the standard notation $\partial_{\tau} X \equiv \dot{X}$ and $\partial_{\sigma} X \equiv X^{\prime}$.

The theory given by the Nambu-Goto action (2.1) has reparametrization invariance, so the canonical Hamiltonian vanishes. Time evolution is completely governed by the constraints, and the Hamiltonian is given by

$$
\mathcal{H}=\lambda\left(P^{2}+T^{2} g g^{00}\right)+\mu\left(P \cdot X^{\prime}\right)
$$

where $\lambda$ and $\mu$ are Lagrange multiplier fields. Their arbitrariness reflects the gauge freedom of the system: setting them to particular values amounts to choosing a particular coordinate system on the worldsheet. The phase-space or Hamiltonian form of the action reads

$$
S_{H}=\int d^{2} \xi(P \dot{X}-\mathcal{H})=\int d^{2} \xi\left(P \cdot\left(\dot{X}-\mu X^{\prime}\right)-\lambda\left(P^{2}+T^{2} g g^{00}\right)\right) .
$$

Integrating out $P$ gives the second order action

$$
S=\frac{1}{2} \int d^{2} \xi \frac{1}{2 \lambda}\left(\left(\dot{X}-\mu X^{\prime}\right)^{2}-4 \lambda^{2} T^{2} g g^{00}\right)
$$

which can be rewritten in the Polyakov form

$$
S_{P}=-\frac{T}{2} \int d^{2} \xi \sqrt{-h} h^{\alpha \beta} \partial_{\alpha} X \cdot \partial_{\beta} X
$$

with the auxiliary metric

$$
h^{\alpha \beta}=\left(\begin{array}{cc}
-1 & \mu \\
\mu & -\mu^{2}+4 \lambda^{2} T^{2}
\end{array}\right) .
$$

The choice $\lambda=\frac{1}{2 T}, \mu=0$ is the conformal gauge. Upon setting $T=0$, the constraints (2.4) reduce to

$$
P^{2}=0, \quad P \cdot X^{\prime}=0,
$$

and the metric degenerates. Note that these are just the constraints of the ambitwistor string, if we replace the prime with a holomorphic derivative $\partial$. It is then possible to rewrite the action (2.10) in terms of an auxiliary field $V^{\alpha}$, defined by

$$
V^{\alpha}=\frac{1}{2 \sqrt{\lambda}}(1,-\mu)
$$

We then obtain the Lindström-Sundborg-Theodoris [29] (LST) tensionless string action

$$
S=\int d^{2} \xi V^{\alpha} V^{\beta} \partial_{\alpha} X \partial_{\beta} X
$$

The equations of motion (EOM) for $V^{\alpha}$ imply that the metric $g_{\alpha \beta}$ has a null eigenvector. Therefore $\operatorname{det} g=0$ which implies that the worldsheet of the tensionless string is a null 
surface, hence the name "null strings". Note that $V^{\alpha}$ transforms as a vector density under worldsheet diffeomorphisms;

$$
\xi^{\alpha} \rightarrow \xi^{\alpha}+\epsilon^{\alpha} \Longrightarrow \delta V^{\alpha}=-V \cdot \partial \epsilon^{\alpha}+\epsilon \cdot \partial V^{\alpha}+\frac{1}{2}(\partial \cdot \epsilon) V^{\alpha}
$$

This transformations maintains the diffeomorphism invariance of the original string, though not its Weyl invariance. Although the metric degenerates in the null string, it is nevertheless possible to obtain its stress energy tensor using the Noether procedure and a local translation transformation. This gives;

$$
T_{\alpha}{ }^{\beta}=V^{\beta} V^{\gamma} \partial_{\gamma} X \cdot \partial_{\alpha} X-\frac{1}{2} \delta_{\alpha}{ }^{\beta} V^{\gamma} V^{\delta} \partial_{\gamma} X \cdot \partial_{\delta} X
$$

By construction, the components of the stress energy tensor are the $T \rightarrow 0$ limit of the constraints (2.4). So there is no change in the fact that gravity is being gauged in the worldsheet, but in the absence of a background non-degenerate metric ${ }^{2}$ the moduli problem becomes more complicated.

In the literature, the commonly studied gauges are

$$
V^{\alpha}=(1,0) \quad \text { or } \quad V^{\alpha}=(0,1)
$$

which we call transverse gauge and dual transverse gauge, respectively, following [23, 30]. The question of whether these gauges can be achieved globally or not has not been studied for the null string. We know however that from the ambitwistor string quantization, the obstruction to gauge fixing precisely furnishes the moduli of the problem. In [25] it is was argued that the vector fields (2.12) have no moduli. While the ambitwistor does have moduli, they are completely fixed by the scattering equations and the resolution of the apparent conflict might lie on this point. We will address the question of moduli for the null string in future work.

\subsection{Constraint algebra}

We review here the construction of $[17,20]$. Imposing the transverse gauge yields the action

$$
S=\frac{1}{2} \int \dot{X}^{2}
$$

which is supplemented by the first-class constraints

$$
\dot{X}^{2}=\dot{X} \cdot X^{\prime}=0
$$

The EOM read

$$
\ddot{X}=0 \text {. }
$$

Together with the constraints, we see that this action describes a collection of massless particles moving at the speed of light, bound together by a constraint forcing their velocities

\footnotetext{
${ }^{2}$ Or equivalently a background complex structure.
} 
to be orthogonal to the string. This last constraint is a remnant of the stringy character of the original tensionful string. The EOM are solved by ${ }^{3}$

$$
X(\tau, \sigma)=Y(\sigma)+\tau P(\sigma)
$$

The mode expansion for each field is defined as

$$
Y(\sigma)=\frac{1}{\sqrt{2 \pi}} \sum_{n \in \mathbb{Z}} y_{n} e^{\mathrm{i} n \sigma}, \quad P(\sigma)=\frac{1}{\sqrt{2 \pi}} \sum_{n \in \mathbb{Z}} p_{n} e^{\mathrm{i} n \sigma}
$$

with Poisson brackets

$$
\left\{y_{n}, p_{m}\right\}_{\mathrm{PB}}=\delta_{n+m, 0} .
$$

The zero modes $y_{0}$ and $p_{0}$ are the centre of mass and momentum of the string. In terms of these, the mode expansion of the constraints read

$$
\begin{aligned}
\dot{X}^{2} & =\frac{1}{2 \pi} \sum_{n}\left(\sum_{m} p_{n+m} p_{-m}\right) e^{i n \sigma}=-\frac{1}{\pi} \sum_{n} M_{n} e^{i n \sigma} \\
\dot{X} \cdot X^{\prime} & =\frac{1}{2 \pi} \sum_{n}\left(\sum_{m} p_{n+m}\left(-\mathrm{i} m y_{-m}-\mathrm{i} m \tau p_{-m}\right)\right) e^{i n \sigma} \\
& =\frac{1}{2 \pi} \sum_{n}\left(L_{n}-i n \tau M_{n}\right) e^{i n \sigma}=0
\end{aligned}
$$

where we have defined the following classical modes for the constraints;

$$
L_{l}=-\mathrm{i} \sum_{n}(l-n) p_{n} \cdot y_{l-n}, \quad M_{l}=-\frac{1}{2} \sum_{n} p_{n} \cdot p_{l-n}
$$

They generate the constraint algebra

$$
\left\{L_{k}, L_{l}\right\}_{\mathrm{PB}}=-\mathrm{i}(k-l) L_{k+l}, \quad\left\{L_{k}, M_{l}\right\}_{\mathrm{PB}}=-\mathrm{i}(l-k) M_{k+l}, \quad\left\{M_{k}, M_{l}\right\}_{\mathrm{PB}}=0,
$$

which is nowadays known as the 2d Galilean Conformal Algebra (GCA) [22, 31]. This algebra can be obtained starting from the two copies of the Virasoro algebra and taking a ultra-relativistic contraction of their generators. In this limit, the small parameter is always accompanied by the tension, so in practice, it is the same as taking the tension to zero. Note that in the GCA there is a single Virasoro left over, which indicates the chiral nature of the null string. This leftover Virasoro cannot be identified with either the left or the right moving Virasoro of the original tensionful string, it is a non-chiral linear combination of both. This symmetry algebra is also the first connection between the null string and the ambitwistor string since the algebra of constraints of the bosonic ambitwistor string is precisely a GCA.

\footnotetext{
${ }^{3}$ We omit the Lorentz index where they can be understood from context.
} 
Geometrically the ultra-relativistic limit of the string is obtained by scaling $\tau \rightarrow \epsilon \tau$ and taking $\epsilon \rightarrow 0$. Thus instead of the two Virasoro algebras which locally generate the diffeomorphisms in time and space we have

$$
L_{n}=\mathrm{i} e^{\mathrm{i} n \sigma}\left(\partial_{\sigma}+\mathrm{i} n \tau \partial_{\tau}\right), \quad M_{n}=\mathrm{i} e^{\mathrm{i} n \sigma} \partial_{\tau}
$$

The relativistic algebra of the string also has another limit, a non-relativistic one. The contracted algebra is isomorphic to the GCA of the ultra-relativistic limit; this is a coincidence that happens in two spacetime dimensions and it is not true in higher dimensions. This limit corresponds to contracting the spatial dimension by $\sigma \rightarrow \epsilon \sigma$ and taking $\epsilon \rightarrow 0$. Locally, there is no difference between taking this limit or the ultra-relativistic one, so it is no surprise that the contracted algebras coincide. In terms of the null string, this contraction can be thought of as corresponding to picking the dual transverse gauge, since the roles of $\tau$ and $\sigma$ are exchanged compared to the ultra-relativistic limit. Here this limit will not play a role so we shall not discuss it further, for more details see [23, 30, 31]. It is also known that the GCA2 algebra is isomorphic to the BMS3 algebra, [32-35]. Asymptotic symmetry algebras also appears in the ambitwistor string when written in coordinates appropriate for a description as having as target space $\mathscr{I}$, the null boundary of Minkowski spacetime [36]. Furthermore, there are four dimensional models which have $\mathscr{I}$ as their target space from the very beginning $[37,38]$. There seems to be an interesting connection between the asymptotic geometry and these models which would be nice to understand.

\subsection{Quantization and spinning string}

The quantization of the null string is done in the standard way by replacing Poisson brackets by commutators $\{,\}_{\mathrm{PB}} \rightarrow-i[$,$] . But this only defines the quantum algebra, which now$ allows certain central terms in the constraint algebra (2.25). In order to define the quantum theory it is also necessary to pick a representation space for this algebra, that is, a choice of vacuum, or equivalently an ordering prescription for the operators. Here, contrary to the tensionful string, there are two inequivalent consistent choices. The first one, discovered in [20], prescribes that the $p_{n}$ 's should be on the right of the $y_{n}$ 's, and accordingly defines the vacuum as the state annihilated by all the $p_{n}$ modes

$$
p_{n}|0\rangle=0, \quad \forall n \in \mathbb{Z} .
$$

This is the so-called Weyl ordering. With this choice of vacuum, it can be shown that the quantized GCA algebra (2.25) does not have a central extension. In particular, the Virasoro subalgebra has zero central charge, implying that Weyl-ordered null strings have no critical dimension [20]. It was proposed in [17, 20] that the spectrum of this theory consists of a mass continuum of free-particles of arbitrary integer higher spins.

This theory is not the ambitwistor string, which has a finite spectrum of massless particles and critical dimension 26 in the bosonic case. But there exists another quantization prescription, which corresponds to pulling to the right all the positive modes of both $y_{n}$ and $p_{n}$, and shifting the negative modes to the left $[17,18]$. This is known as the normal 
ordering prescription. The vacuum is then defined as the state annihilated by the positive modes

$$
p_{n}|0\rangle=y_{n}|0\rangle=0 \quad \text { iff } \quad n>0 .
$$

Let us try to give more substance to the fact that string theory is related to the first theory. In the usual string, the left and right-moving creation/annihilation operators can be defined as

$$
\begin{aligned}
& \alpha_{n}=\frac{1}{2 \sqrt{T}} p_{n}-\mathrm{i} n \sqrt{T} y_{n} \\
& \tilde{\alpha}_{n}=\frac{1}{2 \sqrt{T}} p_{-n}-\mathrm{i} n \sqrt{T} y_{-n} .
\end{aligned}
$$

In the tensionless limit the vacuum conditions $\alpha_{n}|0\rangle=\tilde{\alpha}_{n}|0\rangle=0, \forall n>0$ reduce to $p_{n}|0\rangle=0$ for all $n \in \mathbb{Z}$. However, this limit is singular and it is not at all clear that the modes $\alpha, \tilde{\alpha}$ are defined at all in the limit. Nevertheless, the spectrum in the Weyl quantization is closer to the high-energy string, since it contains an infinite tower of higher spin states.

The normal ordering prescription is closer to what we would get from a chiral CFT. If we imagine that the modes $y_{n}$ and $p_{n}$ are packaged in chiral fields, the usual radial quantization would give the vacuum (2.28). With this prescription, it can be shown, using a variety of methods $[17,18,39]$, that the GCA has a central extension given by a central charge in the Virasoro subalgebra. This gives a critical dimension of 26 for the null string, the same as for the usual string and for the ambitwistor string. Moreover, in this prescription the spectrum is truncated to a finite number of massless modes, which turn out to be the same states as the ones present in the ambitwistor string. The analysis that we present below will also shed some light on why the bosonic (or heterotic) ambitwistor string does not work at the quantum level.

In terms of the spectrum, the key difference between these two quantizations lies in the definition of the quantum operator $L_{0}=\sum_{n} y_{n} \cdot p_{-n}$. In the Weyl ordering, the normal ordering constant turns out to be zero, but in the normal ordering its value is -2 . Recall that $L_{0}$, being the zero mode of $\dot{X} \cdot X^{\prime}$, generates global translations around $\sigma$, so in the latter quantization scheme the spectrum is truncated to operators with angular momentum -2 . This is in essence the same phenomenon that arises in the bosonic ambitwistor string, where the physical states are required to have conformal weight 2 when the ghosts are stripped off. We omit the proofs of these assertions since they can be easily found in the literature, see [17] for instance. We also sketch the calculation for the spinning string below.

The three physical states are $p_{-1}^{\mu} p_{-1}^{\nu}|0\rangle,\left(p_{-1}^{\mu} y_{-1}^{\nu}-y_{-1}^{\mu} p_{-1}^{\nu}\right)|0\rangle$ and $p_{-1}^{\mu} p_{-1}^{\mu}|0\rangle$, they correspond to a graviton, a two-form and a dilaton, as in the bosonic ambitwistor string. Note that using the usual hermitian conjugation, it is evident that these states have zero norm, which points to some inconsistency of the quantum theory. If the bosonic ambitwistor string and the null string are, as we argue below, the same then this inconsistency is to be expected, since from [9] we know that the bosonic ambitwistor string is not target-space diffeomorphism invariant at the quantum level. 
From the same work, we know that the spinning version of the ambitwistor string, with two real fermions on the worldsheet, is target-space diffeomorphism-invariant, which suggest that the spinning null string should not suffer from these issues. Indeed, the authors of $[17,40]$, studied the spinning null string, and showed how it appears classically from the $T \rightarrow 0$ limit of the $\mathcal{N}=(1,1)$ string. The ungauge-fixed LST action [41] is given by

$$
S=\int d^{2} \sigma\left(\left(V^{\alpha} \partial_{\alpha} X^{\mu}+i \Psi^{a \mu} \chi^{a}\right)\left(V^{\beta} \partial_{\beta} X^{\nu}+i \Psi^{b \nu} \chi^{b}\right)+i \Psi^{a, \mu} V^{\alpha} \partial_{\alpha} \Psi_{\mu}^{a}\right),
$$

where the fermions $\Psi^{a \mu}$ transform as densities of weight $-1 / 4$. The index $a$ takes values in $\{1,2\}$ reflecting the two supersymmetries from the tensionful $\mathcal{N}=(1,1)$ string. In the transverse gauge, the bosonic mode expansion is the same as (2.21), while the fermions (in the NS sector) are expanded as

$$
\Psi^{a \mu}(\sigma)=\sum_{r \in \mathbb{Z}+\frac{1}{2}} \psi_{r}^{a \mu} e^{\mathrm{i} r \sigma} .
$$

The constraint algebra of these models was studied thoroughly in $[17,18,42]$. The constraints, written in terms of the canonical momenta, read

$$
\begin{aligned}
P^{2} & =0 \\
P \cdot X^{\prime}+\frac{\mathrm{i}}{2} \sum_{a=1,2} \Psi^{a} \cdot \Psi^{\prime a} & =0 \\
\Psi^{a} \cdot P & =0 \quad(a=1,2) .
\end{aligned}
$$

They are nearly the same as the ambitwistor string. As for the bosonic ambitwistor string, the difference is that in the ambitwistor string the primes in the second constraint are replaced by holomorphic derivatives. Nevertheless the modes algebra is the same for both sets of constraints

$$
\begin{aligned}
{\left[L_{m}, M_{n}\right] } & =(m-n) M_{m+n} \\
{\left[L_{m}, L_{n}\right] } & =(m-n) L_{m+n} \\
{\left[L_{m}, G_{r}^{a}\right] } & =\left(\frac{m}{2}-r\right) G_{m+r}^{a} \\
{\left[G_{r}^{a}, G_{s}^{b}\right]_{+} } & =\delta^{a b} M_{r+s}
\end{aligned}
$$

where $G_{n}^{a}$ are the modes of $\Psi^{a} \cdot P$.

The quantization ambiguity is still present in this case. Choosing t he normal ordering prescription for the bosonic and fermionic modes implies a non-zero normal ordering constant in the constraint $P \cdot X^{\prime}+\frac{i}{2} \Psi^{a} \cdot \Psi^{\prime a}$, which in turn gives a critical dimension $d=10$ in the case of two fermions. ${ }^{4}$ The spectrum in the NS vacuum is given by $p_{-1}|0\rangle$ and $\psi_{-1 / 2}^{a} \psi_{-1 / 2}^{b}|0\rangle$, but after a GSO projection it is truncated to only $\psi_{-1 / 2}^{1} \psi_{-1 / 2}^{2}|0\rangle$. This state, together with the NS-R, R-NS and R-R states, give the spectrum of type II A/B supergravity. As expected these results are the same as for the type II ambitwistor string.

\footnotetext{
${ }^{4}$ Mixed boundary conditions turn out to be inconsistent [17].
} 
What seems to have prevented the authors of [17] from calculating scattering amplitudes for the null string was a lack of knowledge of the vertex operators in their formalism. Here is where the ambitwistor string shines. Since it is written as a CFT, constructing vertex operators is standard, and scattering amplitudes can be easily calculated via OPE's. Models with more supersymmetry were studied in [42], where it is seen that the $\mathcal{N}=4$ model has the same critical dimension as found by Ohmori in [26] for the ambitwistor string with extra SUSY. These connect to the zoo of CHY models [4, 8]. All of this is overwhelming evidence that the ambitwistor string and the null string are the same physical system, provided the correct quantization scheme is chosen for the null string. In the next section we show that how the ambitwistor string can be obtained from the null string.

\section{The ambitwistor string}

We turn now to the ambitwistor string. Our objective here is to describe how it fits into the framework of the null string as introduced above, and thus clarify its relation to the usual tensionful string.

So far, we have avoided discussing a crucial point concerning boundary conditions. Starting from a tensionful string with the standard periodicity $\sigma \simeq \sigma+2 \pi$, we took the null limit and kept this periodicity throughout the process. However, the null string worldsheet has a degenerate metric, so there are no canonical time-like and space-like directions. A time-like direction is needed to perform the usual canonical quantization, and to identify a worldsheet Hamiltonian. It is also not guaranteed from the start that space-like periodicity is not going to clash with the choice of time-like direction. The Schild gauge essentially assumes that the time is given by the direction of $V^{\alpha}$. Given the natural pairing $Z_{\alpha} W^{\alpha}=$ $Z_{0} W^{0}+Z_{1} W^{1}$, a natural choice for the periodic coordinate is in the direction of a $U_{\alpha}$ in the kernel of $V^{\alpha}$. But this solution is not forced upon us and other choices are possible. Indeed the ambitwistor string is one. We will see that the choice $\mu=1$, and the requirement of periodicity along the 1 (or $\sigma$ ) direction will be possible only in the gauge $\lambda=0$.

\subsection{The ambitwistor string action}

The ambitwistor string was originally formulated as a holomorphic CFT in Euclidean signature. In order to make contact with the null string, we Wick-rotate back to Lorentzian signature and write the original action as

$$
S_{A}=\int d^{2} \sigma P \cdot \partial_{-} X
$$

where $\sigma^{ \pm}=\tau \pm \sigma$ and $\partial_{ \pm}=\frac{1}{2}\left(\partial_{\tau} \pm \partial_{\sigma}\right)$. This action is supplemented by the constraints

$$
T_{++}=P \cdot \partial_{+} X=0, \quad P^{2}=0 .
$$

The null string first order action is

$$
S=\int d^{2} \sigma\left(P \cdot \dot{X}-\mu P \cdot X^{\prime}-\lambda P^{2}\right) .
$$


Taking $\mu=1$ and $\lambda=0$ we get the ambitwistor string action, but the slightly different constraints of eq. (2.11). However, using the ambitwistor equation of motion $\partial_{-} X=0$ the triviality

$$
P \cdot X^{\prime}=P \cdot\left(\partial_{-}-\partial_{+}\right) X=P \cdot \partial_{+} X
$$

shows that they are equivalent on-shell. This does not affect the character of the constraints, both the ambitwistor one (3.2), and the null string ones (2.11) are first-class. The relationship between these constraints come from a delicate gauge choice as shown in section 3.4 below.

The importance of the ambitwistor gauge choice is essential. First, choosing the gauge $\mu=1$ effectively picks a background light-cone structure for the worldsheet. In the Wick rotated framework, this is equivalent to a choice of background complex structure, and a choice of a metric. ${ }^{5}$ This is an emergent metric coming purely from the gauge-fixing, the null string has no such non-degenerate metric. Then, let us look at the components of the null string stress tensor (2.15);

$$
\begin{aligned}
& T_{1}^{0}=P \cdot X^{\prime} \\
& T_{0}^{0}=-T_{1}{ }^{1}=2 \lambda P^{2}+\mu P \cdot X^{\prime} \\
& T_{0}{ }^{1}=-\mu\left(4 \lambda P^{2}+\mu P \cdot X^{\prime}\right) .
\end{aligned}
$$

The crucial point is that in the ambitwistor gauge $(\lambda=0, \mu=1)$ and on-shell, the stress tensor of this emergent complex structure coincides with the null string stress-energy tensor. This significantly simplifies finding the moduli for the gauge-fixed null string, as it now coincides with finding the moduli for the effective complex structure. It also enables us to use standard CFT methods to study the null string and compute correlation functions. We expect that a similar mechanism is at work in the other four dimensional twistor strings [43-46], and that they all fit in the framework of null strings. In this regard we mention that in $[47,48]$, a relationship between the four dimensional twistor strings and the null string was already noted.

A more geometrical picture is given by the second-order LST action (2.13). The ambitwistor gauge is singular in the second-order framework since the action is proportional to $\lambda^{-1}$. In order to study it, we fix first an intermediary gauge, where $\mu=1$ and $\lambda$ is unfixed; ${ }^{6}$

$$
V^{\alpha}=\frac{1}{2 \sqrt{\lambda}}(1,-1)
$$

The (partially) gauge-fixed action reads

$$
S=-2 \int d^{2} \xi \frac{1}{\lambda}\left(\partial_{-} X\right)^{2}
$$

Since $\lambda$ has the correct dimension to be a worldsheet loop-counting parameter (like $\hbar$ or $\alpha^{\prime}$ ), the limit $\lambda \rightarrow 0$ appears as a classical limit, where the action is expected to localize

\footnotetext{
${ }^{5}$ More precisely, a conformal class of metrics.

${ }^{6}$ This is similar to what is called the HSZ gauge in Siegel's papers [24, 49], although there it was used in the tensionful string.
} 
on the extremal field configurations, i.e. the classical solutions. In other words, the string partition function $\int \mathcal{D} X e^{-\frac{1}{\lambda} S[X]}$ is independent of $\lambda$, which we can be taken to zero.

\subsection{Residual gauge symmetry}

We follow here an analysis of [27], which we perform in the ambitwistor gauge. In the tensionful string, the leftover symmetries that remain after gauge fixing are the conformal transformations of $z$ and $\bar{z}$. Here the residual symmetries form the Galilean Conformal transformations, generated by the vector field $\epsilon^{\alpha}$ or $\epsilon^{ \pm}=\epsilon^{0} \pm \epsilon^{1}$ with

$$
\left(\epsilon^{+}, \epsilon^{-}\right)=\left(\sigma^{-} f^{\prime}\left(\sigma^{+}\right)+g\left(\sigma^{+}\right), f\left(\sigma^{+}\right)\right)
$$

where, in this section only, prime is the ordinary derivative, not the $\sigma$ derivative. By comparison, conformal transformation are generated by vector fields $\left(\epsilon^{+}, \epsilon^{-}\right)=\left(f\left(\sigma^{+}\right), g\left(\sigma^{-}\right)\right)$. On a function $F\left(\sigma^{-}, \sigma^{+}\right)$, the vector field (3.8) generates the following transformations;

$$
\delta F=\left[\left(\sigma^{-} f^{\prime}\left(\sigma^{+}\right) \partial_{-}+f\left(\sigma^{+}\right) \partial_{+}\right)+g\left(\sigma^{+}\right) \partial_{-}\right] F
$$

from which we define

$$
L(f)=\sigma^{-} f^{\prime}\left(\sigma^{+}\right) \partial_{-}+f\left(\sigma^{+}\right) \partial_{+}, \quad M(g)=g\left(\sigma^{+}\right) \partial_{-}
$$

whose algebra is obtained by decomposing $f$ and $g$ in modes

$$
f=\sum_{n} f_{n} e^{i n \sigma^{+}} \quad g=\sum_{n} g_{n} e^{i n \sigma^{+}}
$$

such that

$$
L(f)=-i \sum_{n} f_{n} L_{n}, \quad M(g)=-i \sum_{n} g_{n} M_{n}
$$

where

$$
L_{n}=i e^{i n \sigma^{+}}\left(\partial_{+}+i n \sigma^{-} \partial_{-}\right), \quad M_{n}=i e^{i n \sigma^{+}} \partial_{-} .
$$

They satisfy the following commutation relations;

$$
\left[L_{n}, L_{m}\right]=(n-m) L_{n+m}, \quad\left[L_{n}, M_{m}\right]=(n-m) M_{n+m}, \quad\left[M_{n}, M_{m}\right]=0 .
$$

It seems that in the ambitwistor gauge we have lost the periodicity of the original null string. In the next section we shall see that this is merely an artifact of this gauge choice.

\subsection{Equations of motion in the second order form}

Using the second order action in chiral gauge (3.7), the equations of motion are

$$
\partial_{-} \partial_{-} X=0
$$

subject to the constraints

$$
\begin{aligned}
\left(\partial_{-} X\right)^{2} & =0 \\
\partial_{-} X \cdot \partial_{\sigma} X & =0
\end{aligned}
$$


where the constraint (3.16) is the EOM for $V^{\alpha}$, just like in the standard string, where the EOM for the auxiliary metric condition gives the Virasoro constraints $T_{\alpha \beta}=0$. The EOM are solved by

$$
X\left(\sigma^{-}, \sigma^{+}\right)=Y\left(\sigma^{+}\right)+\sigma^{-} K\left(\sigma^{+}\right)
$$

with mode expansion

$$
Y\left(\sigma^{+}\right)=\frac{1}{\sqrt{2 \pi}} \sum_{n} y_{n} e^{-\mathrm{i} n \sigma^{+}}, \quad K\left(\sigma^{+}\right)=\frac{1}{\sqrt{2 \pi}} \sum_{n} k_{n} e^{-\mathrm{i} n \sigma^{+}} .
$$

It seems that the solution (3.18) breaks the string's periodicity $\sigma_{ \pm} \simeq \sigma_{ \pm} \pm 2 \pi$. In order to restore it we impose the periodicity conditions

$$
\begin{aligned}
& K\left(\sigma^{+}+2 \pi\right)=K\left(\sigma^{+}\right) \\
& Y\left(\sigma^{+}+2 \pi\right) \simeq Y\left(\sigma^{+}\right)+2 \pi K\left(\sigma^{+}\right),
\end{aligned}
$$

or in terms of the modes $y_{n} \simeq y_{n}+2 \pi k_{n}$. This condition is consistent with the Poisson brackets

$$
\begin{aligned}
& \left\{y_{n}, y_{m}\right\} \simeq\left\{y_{n}+2 \pi k_{n}, y_{m}+2 \pi k_{m}\right\}=0 \\
& \left\{y_{n}, k_{m}\right\} \simeq\left\{y_{n}+2 \pi k_{n}, k_{m}\right\}=\delta_{m+n, 0} .
\end{aligned}
$$

One might worry about the constraint algebra, and indeed at first sight it seems that there will be problems in defining the modes for $\partial_{-} X \cdot X^{\prime}$. Ultimately, these issues stem from a tension between the periodicity in the gauge $\mu=1$ and the singularity of the gauge $\lambda=0$ that we are trying to impose in the second-order action. Note that the canonical momentum depends on $\lambda$

$$
P=\frac{1}{\lambda} \partial_{-} X=\frac{1}{\lambda} K
$$

so, in order to have a finite canonical momenta when $\lambda \rightarrow 0$ we need to rescale the modes of $K$ by $\lambda$. Therefore, this rescaling leaves the $P^{2}$ constraint invariant, but does affect the $P \cdot X^{\prime}$ constraint

$$
\frac{1}{\lambda} \partial_{-} X \cdot X^{\prime}=P \cdot\left(Y^{\prime}-K-\sigma K^{\prime}\right)=P \cdot\left(Y^{\prime}-\lambda P-\lambda \sigma P^{\prime}\right) \rightarrow P \cdot Y^{\prime}
$$

where all the constraints are evaluated at $\tau=0$ for simplicity. We see that in the limit we recover the first-order constraints, as expected, and that the periodicity condition on the modes $y_{n}$ disappears. This shows that to work with the more geometric second-order Lagrangian we should be very careful in order not to run into inconsistencies. Next we rephrase the same question in the first order form, where the subtleties are absent.

\subsection{Equations of motion in the first order form}

Using the first order action in the $\mu=1$ gauge, with $\lambda$ yet unfixed, the EOM for $P$ and $X$ read

$$
\partial_{-} X=2 \lambda P, \quad \partial_{-} P=0
$$


still subject to the constraints eq. (2.11). The EOM are solved by

$$
P=P\left(\sigma^{+}\right), \quad X=Y\left(\sigma^{+}\right)+2 \lambda \sigma^{-} P\left(\sigma^{+}\right)
$$

with mode expansion

$$
Y\left(\sigma^{+}\right)=\frac{1}{\sqrt{2 \pi}} \sum_{n} y_{n} e^{-\mathrm{i} n \sigma^{+}}, \quad P\left(\sigma^{+}\right)=\frac{1}{\sqrt{2 \pi}} \sum_{n} p_{n} e^{-\mathrm{i} n \sigma^{+}} .
$$

It seems a priori that the solution (3.27) breaks the periodicity $\sigma_{ \pm} \simeq \sigma_{ \pm} \pm 2 \pi$. But setting $\lambda=0$, the $\sigma$-periodicity is restored and the mode expansion of the $P X^{\prime}$ constraint simplifies to the following;

$$
\left.P \cdot X^{\prime}\right|_{\lambda=0}=P\left(\sigma^{+}\right) \cdot \partial_{\sigma} Y\left(\sigma^{+}\right)=-\frac{\mathrm{i}}{2 \pi} \sum_{n, m} n y_{-m} \cdot p_{n+m} e^{-\mathrm{i} n \sigma^{+}}=\frac{1}{2 \pi} \sum L_{n} e^{-i n \sigma^{+}}
$$

( $P^{2}$ does not change).

We give now a somewhat heuristic argument to support the idea that the singular gauge $\lambda=0$ really allows one gauge the stress-tensor of a chiral CFT and not just the $P \cdot X^{\prime}$ constraint. For arbitrary $\lambda$, it is not hard to see that

$$
P \cdot \partial_{+} X \propto \sum_{n}\left(L_{n}-i n \lambda \sigma^{-} M_{n}\right) e^{-i n \sigma^{+}} .
$$

with $L_{n}$ and $M_{n}$ defined as in eq. (2.24). Hence, at $\lambda=0$, we see that the $L_{n}$ 's, which are the modes of the null string constraints really do coincide with the modes of the stresstensor.

On the other hand, it is evident, from looking at the Lie algebra representation of the $L$ and $M$ 's in eq. (3.13), that the combination $L_{n}^{\prime} \equiv\left(L_{n}-i n \sigma^{-} M_{n}\right)=-\mathrm{i} e^{-i n \sigma^{+}} \partial_{+}$are the generators of conformal transformations on $\sigma^{-}$. Moreover, the $L_{n}^{\prime}$ generator obey the same algebra with the $M_{n}$ 's, so this is an automorphism of the GCA algebra. ${ }^{7}$ So it seems that the singular gauge choice $\lambda=0$ automatically enforces that the constraint algebra of the tensionless string does become that of the holomorphic stress-tensor of a chiral CFT.

\section{Relation to tensionful strings}

\subsection{The bosonic string}

At the classical level, the relationship between the ambitwistor string and the tensionful string is straightforward. As we have shown above, the classical ambitwistor string is nothing else than the tensionless limit of the usual string in an unusual gauge. It is at the quantum level that the relationship becomes interesting. This is due to the non-standard (from the tensionful string point of view) choice of vacuum for the ambitwistor string. Recall that the ambitwistor vacuum is defined as the state annihilated by the positive

\footnotetext{
${ }^{7}$ We are grateful to Blagoje Oblak for a discussion on this point.
} 
modes of $P$ and $X$, see (2.28). To make contact with the usual string, recall the definition of the modes

$$
\begin{aligned}
& \alpha_{n}=\frac{1}{2 \sqrt{T}} p_{n}-\mathrm{i} n \sqrt{T} y_{n} \\
& \tilde{\alpha}_{n}=\frac{1}{2 \sqrt{T}} p_{-n}-\mathrm{i} n \sqrt{T} y_{-n}
\end{aligned}
$$

which inherit the commutation relations

$$
\left[\alpha_{n}, \alpha_{m}\right]=\left[\tilde{\alpha}_{n}, \tilde{\alpha}_{m}\right]=n \delta_{m+n, 0}
$$

from the canonical ones $\left[y_{n}, p_{m}\right]=i \delta_{n+m, 0}$. In terms of these modes, the ambitwistor vacuum obeys

$$
\alpha_{n}|0\rangle_{A}=\tilde{\alpha}_{-n}|0\rangle_{A}=0, \quad \forall n>0,
$$

in stark contrast to the string theory vacuum, defined by

$$
\alpha_{n}|0\rangle_{S}=\tilde{\alpha}_{n}|0\rangle_{S}=0, \quad \forall n>0 .
$$

The alternative choice of vacuum (4.3) for the tensionful string was briefly considered in [39]. The authors noted that, although the vacuum is not BRST-invariant, there are physical massless states. Unfortunately, these have negative norm. Interestingly this choice of vacuum changes the normal ordering constant of the $\tilde{\mathcal{L}}_{0}$ mode from -1 to 1 . This is due to the fact that a consistent BRST quantization of this vacuum requires that $\tilde{c}_{-m}|0\rangle_{A}=0$ for all $n>0$, in contrast to the right-handed condition $c_{m}|0\rangle_{A}=0$ for $m>0$. Recall the definition of the Virasoro generators;

$$
\begin{aligned}
& \mathcal{L}_{m}=\frac{1}{2} \sum_{n}: \alpha_{m-n} \cdot \alpha_{n}:+\sum_{n}(2 m-n): b_{n} c_{m-n}:+a \delta_{m, 0} \\
& \tilde{\mathcal{L}}_{m}=\frac{1}{2} \sum_{n}: \tilde{\alpha}_{m-n} \cdot \tilde{\alpha}_{n}:+\sum_{n}(2 m-n): \tilde{b}_{n} \tilde{c}_{m-n}:+\tilde{a} \delta_{m, 0} .
\end{aligned}
$$

The normal ordering constant is obtained by standard methods which we reproduce here to highlight the difference with the usual string. Starting from $\left(\mathcal{L}_{0}-a\right)|0\rangle_{A}=0$ and $\left(\tilde{\mathcal{L}}_{0}-\tilde{a}\right)|0\rangle_{A}=0$, we use

$$
\begin{aligned}
2 \mathcal{L}_{0}|0\rangle_{A}=\left(\mathcal{L}_{1} \mathcal{L}_{-1}-\mathcal{L}_{-1} \mathcal{L}_{1}\right)|0\rangle_{A}=\mathcal{L}_{1} \mathcal{L}_{-1}|0\rangle_{A} & =-\left(2 b_{0} c_{1}\right)\left(b_{-1} c_{0}\right)|0\rangle_{A} \\
& =-|0\rangle_{A} \\
2 \tilde{\mathcal{L}}_{0}|0\rangle_{A}=\left(\tilde{\mathcal{L}}_{1} \tilde{\mathcal{L}}_{-1}-\tilde{\mathcal{L}}_{-1} \tilde{\mathcal{L}}_{1}\right)|0\rangle_{A}=-\tilde{\mathcal{L}}_{-1} \tilde{\mathcal{L}}_{1}|0\rangle_{A} & =\left(2 \tilde{b}_{0} \tilde{c}_{-1}\right)\left(\tilde{b}_{1} \tilde{c}_{0}\right)|0\rangle_{A} \\
& =+|0\rangle_{A} .
\end{aligned}
$$

Therefore, the normal ordering ambiguity of the operator $\mathcal{L}_{0}+\tilde{\mathcal{L}}_{0}$, which contains the massshell condition, is transferred to the angular momentum operator $\mathcal{L}_{0}-\tilde{\mathcal{L}}_{0}$. Physical states are then restricted to those with angular momentum 2 and the spectrum is truncated. Contrary to the null string the spectrum is not all massless, there are two massive states 
with the same, but opposite mass proportional to the tension $T .{ }^{8}$ Consistency of the quantization procedure also requires that the vacuum obeys

$$
\mathcal{L}_{n}|0\rangle_{A}=\tilde{\mathcal{L}}_{-n}|0\rangle_{A}=0, \quad \forall n>0,
$$

this, in particular, effectively exchanges the roles of translations and special conformal transformations in the left-handed part of the conformal algebra. That is, we are picking different representation spaces for the right-handed Virasoro and for the left-handed Virasoro.

Going one step further we can define the modes

$$
\begin{aligned}
L_{n} & =\left(\mathcal{L}_{n}-\tilde{\mathcal{L}}_{-n}\right)=-\mathrm{i} \sum_{k} k p_{n-k} \cdot y_{k}-2 \delta_{n, 0} \\
M_{n} & =T\left(\mathcal{L}_{n}+\tilde{\mathcal{L}}_{-n}\right)=\frac{1}{2} \sum_{k}\left(\frac{1}{2} p_{n-k} \cdot p_{k}+2 T^{2}(k-n) k y_{n-k} \cdot y_{k}\right) .
\end{aligned}
$$

In the $T \rightarrow 0$ limit they coincide with the constraint modes of the ambitwistor string, including the normal ordering constant. This change of sign of the zero mode constant was also noted in $[24,50]$, where it was attributed to a 'change in boundary conditions' for the bosonic string $X$ field. As explained above our point of view is that this is the result of a twisted quantization schemes for the string.

We further note that the ambitwistor vacuum $|0\rangle_{A}$ and the string theory vacuum $|0\rangle_{S}$ are not related by a Bogolioubov transformation, they give inequivalent quantizations of the classical string. That is, these vacua live in different, unitarily inequivalent, Hilbert spaces. This implies that the quantum ambitwistor string is not a subsector of the usual string in any natural way. This also explains why the ambitwistor string does not come from the $T \rightarrow \infty$ limit of the string (field theory or $\alpha^{\prime} \rightarrow 0$ limit), since it is in fact the null string in disguise. One last thing to note is that the combination of modes (4.10) corresponds to the well-defined modes in the ultrarelativistic limit of the Virasoro algebra, which is equivalent to the tensionless limit of the algebra [23,30].

To close this section we mention that the above alternative quantization of the string can be achieved by a deformation of the ambitwistor string. We keep the chiral action (3.1), but we deform the $P^{2}$ constraint to

$$
\mathcal{H}=\frac{1}{2}\left(P^{2}+(\partial X)^{2}\right) .
$$

Using the free OPEs, it is easy to see that the algebra of constraints is modified to

$$
\begin{aligned}
\mathcal{T}(z) \mathcal{T}(w) & \simeq \frac{2 \mathcal{T}}{(z-w)^{2}}+\frac{\partial \mathcal{T}}{(z-w)} \\
\mathcal{T}(z) \mathcal{H}(w) & \simeq \frac{2 \mathcal{H}}{(z-w)^{2}}+\frac{\partial \mathcal{H}}{(z-w)} \\
\mathcal{H}(z) \mathcal{H}(w) & \simeq \frac{2 \mathcal{T}}{(z-w)^{2}}+\frac{\partial \mathcal{T}}{(z-w)}
\end{aligned}
$$

\footnotetext{
${ }^{8}$ This was also noted in [39, 50].
} 
where $\mathcal{T}=-P \cdot \partial X$ is the stress-energy tensor. The massless sector consists of the BRST closed vertex operators

$$
\begin{aligned}
& U=c \tilde{c} \epsilon_{\mu \nu}\left(P^{\mu} P^{\nu}-\partial X^{\mu} \partial X^{\nu}\right) e^{\mathrm{i} k \cdot X} \\
& B=c \tilde{c} \epsilon_{\mu \nu} P^{[\mu} \partial X^{\nu]} e^{\mathrm{i} k \cdot X}
\end{aligned}
$$

plus an scalar operator. In lightcone gauge it is easy to see that these correspond to a graviton, b-field and dilaton. The fields $c$ and $\tilde{c}$ are the ghosts for the constraints, see [6]. Contrary to the original bosonic ambitwistor string the cohomology now allows for massive states. There are two massive spin two states given by

$$
V=c \tilde{c} \epsilon_{\mu \nu}\left(P^{\mu} P^{\nu}+\partial X^{\mu} \partial X^{\nu} \pm P^{(\mu} \partial X^{\nu)}\right) e^{\mathrm{i} k \cdot X}
$$

with masses $k^{2}=\mp 4$ in our conventions as well as two massive scalars also with masses $k^{2}=\mp 4$. These can be interpreted as the trace part of the above massive spin two states. Besides being a realization of the alternative quantization of the string, this is very reminiscent of the HSZ theory [49-51], thought the spectrum of massive scalars in [51] is double what we've found. Nevertheless, above CFT might help in calculating higher-point amplitudes in this theory. The tension $T$ might be restored by dimensional analysis, and in the $T \rightarrow 0$ limit the two massive spin two states become massless and indistinguishable from the graviton vertex operator. In this limit the model reduces to the original ambitwistor string.

\subsection{The spinning string}

The bosonic string in the alternative quantization scheme (4.3) has negative normed states which are the same as the ones found in the null string. The next step is then to add supersymmetry and see if the resulting model has only positive normed states in analogy with the null string. We will see that this is indeed the case, though the resulting quantization procedure looks strange from the original worldsheet perspective. ${ }^{9}$

Classically the supersymmetry is the same as the usual $(1,1)$ string, that is, the fermionic generators are

$$
\mathcal{G}_{r}=\sum \alpha_{n} \cdot \psi_{r-n}, \quad \tilde{\mathcal{G}}_{r}=\sum \tilde{\alpha}_{n} \cdot \tilde{\psi}_{r-n} .
$$

To have a consistent BRST quantization their action on the vacuum must be different form the usual string, in particular $\tilde{\psi}_{r}|0\rangle_{A}=0$ for all $r>-\frac{1}{2}$. This has a cascading effect for the left-handed fields. The bosonic ghost vacuum now obeys $\tilde{\gamma}_{-n}|0\rangle_{A}=0$ for $n>0$ which contributes $+\frac{1}{2}$ to the normal ordering constant of $\tilde{\mathcal{L}}_{0}$. The end result is that all the left-handed fields have their quantization flipped, that is, their positive modes now annihilate the vacuum, and the spectrum is truncated to states with angular momentum 1. This is analogous to the bosonic case, but now the GSO projected physical states are built

\footnotetext{
${ }^{9}$ Instead of the requirement that right movers are holomorphic around the origin and left movers antiholomorphic around the origin, the twisted quantization requires right movers to be holomorphic around the origin and left movers to be antiholomorphic around infinity.
} 
out of the fermions, $\psi_{-1 / 2} \tilde{\psi}_{1 / 2}|k\rangle_{A}$, instead of the modes of $X$. Once gauge-invariances are taken into account ${ }^{10}$ the physical states have positive norm. This reflects the situation for the null spinning string, but here the tension has been kept finite. It is interesting to note that, like in the bosonic case, there are possible massive states, of the form $\psi \psi$ and $\tilde{\psi} \tilde{\psi}$. However, they are projected out by the GSO projection. So, even at finite $T$ the only physical states in the spectrum are massless.

It is easy to see that the algebra of constraints limits nicely into the ambitwistor string constraint algebra. Define the modes

$$
\begin{aligned}
L_{n} & =\left(\mathcal{L}_{n}-\tilde{\mathcal{L}}_{-n}\right), & M_{n} & =T\left(\mathcal{L}_{n}+\tilde{\mathcal{L}}_{-n}\right), \\
G_{r} & =\sqrt{T} \mathcal{G}_{n}, & \tilde{G}_{r} & =\sqrt{T} \tilde{\mathcal{G}}_{-n} .
\end{aligned}
$$

The bosonic part of the algebra mimics what happens in the bosonic string. The relevant fermionic anticommutators are

$$
\begin{aligned}
& \left\{G_{r}, G_{s}\right\}=\left\{\tilde{G}_{r}, \tilde{G}_{s}\right\}=M_{r+s} \\
& \left\{G_{r}, \tilde{G}_{s}\right\}=T L_{r+s} .
\end{aligned}
$$

When $T=0$, this algebra is isomorphic to that of the spinning ambitwistor string [6], and also coincide with the commutators found in [17] for the worldsheet supersymmetrization of the null string. As seen in the bosonic case, the tensionless limit mixes left and righthanded generators in a non-trivial way. We note that this algebra does not coincide with a supersymmetrization of the GCA algebra used in [52]. In our case, we are interested in the $T \rightarrow 0$ limit of the algebra, which corresponds to the ultra-relativistic limit of the bosonic part of the string, while in [52], the limit studied also involves contracting the odd sector.

In the purely bosonic case the algebras obtained from these two limits are isomorphic. In the supersymmetric case there seems to be some freedom in how the fermionic generators are rescaled and combined in order to get finite, non-trivial generators. Clearly, the scalings above are the correct ones in order to reproduce the spinning tensionless string, but it would be interesting to study if the other possible scalings give interesting theories. This limit can also be taking at the quantum level in a similar way to the bosonic case, and the central charge is seen to still vanishes only in ten dimensions.

We close this section by noting that the construction of $[53]^{11}$ appears as a hybrid formulation, somehow close to Fairlie \& Robert's original paper [55]. The string integrand is computed at finite tension, but the nearest neighbour interaction term $T^{2} X^{\prime 2}$ is dropped in the constraint eq. (2.4). It remains to be understood how this fits exactly in the rich network of theories that were discussed here and in the aforementioned references.

\section{Discussion}

We showed that the ambitwistor string is only related to the usual string at the classical level, being a way of describing the $T \rightarrow 0$ limit of the string, i.e. the null string. This

\footnotetext{
${ }^{10}$ This is most easily done by going to lightcone gauge.

${ }^{11}$ Some observations about the connection between integration by parts in string theory and the scattering equations were also made in [54].
} 
also explains why the scattering equations, a feature appearing in the high energy limit of strings, also appears in a crucial way in the ambitwistor string. Though a more complete treatment of the moduli problem for the null string is left for future work. It is at the quantum level that these theories differ in a profound way. The quantization procedures for the two theories are inequivalent and there is no canonical way of relating the vacuum in one theory to the vacuum in the other. Nevertheless, one might still hope to find $\alpha^{\prime}$ corrections to the ambitwistor string keeping in mind the issues addressed in this paper. There are also other questions to address in future works; like other possible null strings and their ambitwistor description, including the tensionless limit of heterotic and open strings, and especially the construction of the measure on the moduli space.

\section{Acknowledgments}

We would like to thank Blagoje Oblak, Lionel Mason, Nima Doroud, David Skinner, Bo Sundborg for interesting discussions and comments and Yvonne Geyer for comments on the draft. The work of ED is supported by EPSRC grant EP/M018911/1, the work of PT is supported by STFC grant ST/L000385/1 and Queens' College Cambridge Postdoctoral Research Associateship.

Open Access. This article is distributed under the terms of the Creative Commons Attribution License (CC-BY 4.0), which permits any use, distribution and reproduction in any medium, provided the original author(s) and source are credited.

\section{References}

[1] F. Cachazo, S. He and E.Y. Yuan, Scattering of Massless Particles: Scalars, Gluons and Gravitons, JHEP 07 (2014) 033 [arXiv:1309.0885] [INSPIRE].

[2] F. Cachazo, S. He and E.Y. Yuan, Scattering of Massless Particles in Arbitrary Dimensions, Phys. Rev. Lett. 113 (2014) 171601 [arXiv:1307.2199] [InSPIRE].

[3] F. Cachazo, S. He and E.Y. Yuan, Scattering equations and Kawai-Lewellen-Tye orthogonality, Phys. Rev. D 90 (2014) 065001 [arXiv:1306.6575] [INSPIRE].

[4] F. Cachazo, S. He and E.Y. Yuan, Scattering Equations and Matrices: From Einstein To Yang-Mills, DBI and NLSM, JHEP 07 (2015) 149 [arXiv:1412.3479] [INSPIRE].

[5] F. Cachazo, S. He and E.Y. Yuan, Einstein- Yang-Mills Scattering Amplitudes From Scattering Equations, JHEP 01 (2015) 121 [arXiv: 1409.8256] [INSPIRE].

[6] L. Mason and D. Skinner, Ambitwistor strings and the scattering equations, JHEP 07 (2014) 048 [arXiv: 1311.2564] [INSPIRE].

[7] N. Berkovits, Infinite Tension Limit of the Pure Spinor Superstring, JHEP 03 (2014) 017 [arXiv: 1311.4156] [INSPIRE].

[8] E. Casali, Y. Geyer, L. Mason, R. Monteiro and K.A. Roehrig, New Ambitwistor String Theories, JHEP 11 (2015) 038 [arXiv:1506.08771] [INSPIRE].

[9] T. Adamo, E. Casali and D. Skinner, A Worldsheet Theory for Supergravity, JHEP 02 (2015) 116 [arXiv:1409.5656] [INSPIRE]. 
[10] T. Adamo and E. Casali, Scattering equations, supergravity integrands and pure spinors, JHEP 05 (2015) 120 [arXiv: 1502.06826] [INSPIRE].

[11] T. Adamo, E. Casali and D. Skinner, Ambitwistor strings and the scattering equations at one loop, JHEP 04 (2014) 104 [arXiv:1312.3828] [INSPIRE].

[12] E. Casali and P. Tourkine, Infrared behaviour of the one-loop scattering equations and supergravity integrands, JHEP 04 (2015) 013 [arXiv: 1412.3787] [INSPIRE].

[13] Y. Geyer, L. Mason, R. Monteiro and P. Tourkine, Loop Integrands for Scattering Amplitudes from the Riemann Sphere, Phys. Rev. Lett. 115 (2015) 121603 [arXiv:1507.00321] [INSPIRE].

[14] Y. Geyer, L. Mason, R. Monteiro and P. Tourkine, One-loop amplitudes on the Riemann sphere, JHEP 03 (2016) 114 [arXiv:1511.06315] [INSPIRE].

[15] D. Amati, M. Ciafaloni and G. Veneziano, Superstring Collisions at Planckian Energies, Phys. Lett. B 197 (1987) 81 [inSPIRE].

[16] D.J. Gross and P.F. Mende, String Theory Beyond the Planck Scale, Nucl. Phys. B 303 (1988) 407 [INSPIRE].

[17] J. Gamboa, C. Ramirez and M. Ruiz-Altaba, Null Spinning Strings, Nucl. Phys. B 338 (1990) 143 [INSPIRE].

[18] J. Gamboa, C. Ramirez and M. Ruiz-Altaba, Quantum Null (Super)Strings, Phys. Lett. B 225 (1989) 335 [INSPIRE].

[19] A. Schild, Classical Null Strings, Phys. Rev. D 16 (1977) 1722 [INSPIRE].

[20] F. Lizzi, B. Rai, G. Sparano and A. Srivastava, Quantization of the Null String and Absence of Critical Dimensions, Phys. Lett. B 182 (1986) 326 [INSPIRE].

[21] D.J. Gross, High-Energy Symmetries of String Theory, Phys. Rev. Lett. 60 (1988) 1229 [INSPIRE].

[22] A. Bagchi and R. Gopakumar, Galilean Conformal Algebras and AdS/CFT, JHEP 07 (2009) 037 [arXiv:0902.1385] [INSPIRE].

[23] A. Bagchi, Tensionless Strings and Galilean Conformal Algebra, JHEP 05 (2013) 141 [arXiv:1303.0291] [INSPIRE].

[24] W. Siegel, Amplitudes for left-handed strings, arXiv:1512.02569 [INSPIRE].

[25] B. Sundborg, Strongly topological interactions of tensionless strings, hep-th/9405195 [INSPIRE].

[26] K. Ohmori, Worldsheet Geometries of Ambitwistor String, JHEP 06 (2015) 075 [arXiv: 1504.02675] [INSPIRE].

[27] J. Isberg, U. Lindström, B. Sundborg and G. Theodoridis, Classical and quantized tensionless strings, Nucl. Phys. B 411 (1994) 122 [hep-th/9307108] [INSPIRE].

[28] A. Karlhede and U. Lindström, The Classical Bosonic String in the Zero Tension Limit, Class. Quant. Grav. 3 (1986) L73 [InSPIRE].

[29] U. Lindström, B. Sundborg and G. Theodoridis, The Zero tension limit of the superstring, Phys. Lett. B 253 (1991) 319 [INSPIRE].

[30] A. Bagchi, S. Chakrabortty and P. Parekh, Tensionless Strings from Worldsheet Symmetries, JHEP 01 (2016) 158 [arXiv: 1507.04361] [INSPIRE]. 
[31] A. Bagchi, R. Gopakumar, I. Mandal and A. Miwa, GCA in 2d, JHEP 08 (2010) 004 [arXiv:0912.1090] [INSPIRE].

[32] A. Campoleoni, H.A. Gonzalez, B. Oblak and M. Riegler, BMS Modules in Three Dimensions, in proceedings of the International Workshop on Higher Spin Gauge Theories, Singapore, November 4-6 2015 [Int. J. Mod. Phys. A 31 (2016) 1650068] [arXiv: 1603.03812] [INSPIRE].

[33] G. Barnich and B. Oblak, Notes on the BMS group in three dimensions: I. Induced representations, JHEP 06 (2014) 129 [arXiv:1403.5803] [INSPIRE].

[34] G. Barnich, L. Donnay, J. Matulich and R. Troncoso, Asymptotic symmetries and dynamics of three-dimensional flat supergravity, JHEP 08 (2014) 071 [arXiv:1407.4275] [INSPIRE].

[35] G. Barnich and C. Troessaert, Aspects of the BMS/CFT correspondence, JHEP 05 (2010) 062 [arXiv: 1001.1541] [INSPIRE].

[36] Y. Geyer, A.E. Lipstein and L. Mason, Ambitwistor strings at null infinity and (subleading) soft limits, Class. Quant. Grav. 32 (2015) 055003 [arXiv:1406.1462] [INSPIRE].

[37] T. Adamo, E. Casali and D. Skinner, Perturbative gravity at null infinity, Class. Quant. Grav. 31 (2014) 225008 [arXiv: 1405.5122] [INSPIRE].

[38] T. Adamo and E. Casali, Perturbative gauge theory at null infinity, Phys. Rev. D 91 (2015) 125022 [arXiv: 1504.02304] [INSPIRE].

[39] S. Hwang, R. Marnelius and P. Saltsidis, A general BRST approach to string theories with zeta function regularizations, J. Math. Phys. 40 (1999) 4639 [hep-th/9804003] [INSPIRE].

[40] J. Barcelos-Neto, C. Ramirez and M. Ruiz-Altaba, Phase Space Lagrangians for Null Spinning Strings, Z. Phys. C 47 (1990) 241 [INSPIRE].

[41] U. Lindström, B. Sundborg and G. Theodoridis, The Zero tension limit of the spinning string, Phys. Lett. B 258 (1991) 331 [INSPIRE].

[42] P. Saltsidis, Hamiltonian BRST quantization of the conformal spinning string, Nucl. Phys. B 446 (1995) 286 [hep-th/9503062] [INSPIRE].

[43] E. Witten, Perturbative gauge theory as a string theory in twistor space, Commun. Math. Phys. 252 (2004) 189 [hep-th/0312171] [INSPIRE].

[44] N. Berkovits, An Alternative string theory in twistor space for $N=4$ super Yang-Mills, Phys. Rev. Lett. 93 (2004) 011601 [hep-th/0402045] [INSPIRE].

[45] D. Skinner, Twistor Strings for $N=8$ Supergravity, arXiv:1301.0868 [INSPIRE].

[46] Y. Geyer, A.E. Lipstein and L.J. Mason, Ambitwistor Strings in Four Dimensions, Phys. Rev. Lett. 113 (2014) 081602 [arXiv:1404.6219] [INSPIRE].

[47] W. Siegel, Untwisting the twistor superstring, hep-th/0404255 [INSPIRE].

[48] I. Bandos, Twistor/ambitwistor strings and null-superstrings in spacetime of D =4, 10 and 11 dimensions, JHEP 09 (2014) 086 [arXiv: 1404.1299] [INSPIRE].

[49] O. Hohm, W. Siegel and B. Zwiebach, Doubled $\alpha^{\prime}$-geometry, JHEP 02 (2014) 065 [arXiv: 1306.2970] [INSPIRE].

[50] Y.-t. Huang, W. Siegel and E.Y. Yuan, Factorization of Chiral String Amplitudes, JHEP 09 (2016) 101 [arXiv: 1603.02588] [INSPIRE]. 
[51] O. Hohm, U. Naseer and B. Zwiebach, On the curious spectrum of duality invariant higher-derivative gravity, JHEP 08 (2016) 173 [arXiv: 1607.01784] [INSPIRE].

[52] I. Mandal, Supersymmetric Extension of GCA in 2d, JHEP 11 (2010) 018 [arXiv: 1003.0209] [INSPIRE].

[53] N.E.J. Bjerrum-Bohr, P.H. Damgaard, P. Tourkine and P. Vanhove, Scattering Equations and String Theory Amplitudes, Phys. Rev. D 90 (2014) 106002 [arXiv: 1403.4553] [INSPIRE].

[54] H. Gómez and E.Y. Yuan, N-point tree-level scattering amplitude in the new Berkovits' string, JHEP 04 (2014) 046 [arXiv: 1312.5485] [INSPIRE].

[55] D.B. Fairlie and D.E. Roberts, Dual Models without Tachyons - a New Approach, PRINT-72-2440 [INSPIRE]. 